\title{
ON NON-COMMUTATIVE FORMAL DEFORMATIONS OF COHERENT SHEAVES ON AN ALGEBRAIC VARIETY
}

\author{
YUJIRO KAWAMATA
}

\section{Contents}

1. Introduction

2. Non-commutative deformations and DG algebra

3. Review on $A^{\infty}$-algebra

4. Description using $A^{\infty}$-structure

5. 1-pointed versus $r$-pointed deformations

6. Remark on universal extensions 13

7. Examples 14

References 23

\begin{abstract}
We review the theory of non-commutative deformations of sheaves and describe a versal deformation by using an $A^{\infty}$-algebra and the change of differentials of an injective resolution. We give some explicit non-trivial examples. 14D15, 14F05
\end{abstract}

\section{INTRODUCTION}

We consider non-commutative deformations of sheaves on an algebraic variety in this paper. We consider also multi-pointed deformations, and give some non-trivial examples. The point is that such deformation theory is more natural than the commutative ones as long as we consider infinitesimal deformations.

Let $F$ be a coherent sheaf on an algebraic variety $X$ defined over a field $k$ such that the support of $F$ is proper. We can consider a moduli space $M$ which parametrizes flat deformations of $F$. The infinitesimal study of $M$ is to investigate the completed local ring $\hat{\mathcal{O}}_{M,[F]}$ at a point corresponding to $F$. The tangent space of $M$ at $[F]$ is isomorphic to $\operatorname{Ext}^{1}(F, F)$, and the singularitiy at $[F]$ is described by using the obstruction space $\operatorname{Ext}^{2}(F, F)$. Thus we can write

$$
\hat{\mathcal{O}}_{M,[F]}=k\left[\left[\operatorname{Ext}^{1}(F, F)^{*}\right]\right] /\left(\operatorname{Ext}^{2}(F, F)^{*}\right)
$$

where ${ }^{*}$ denotes the dual vector space, $k\left[\left[\operatorname{Ext}^{1}(F, F)^{*}\right]\right]$ is the completed symmetric tensor algebra of $\operatorname{Ext}^{1}(F, F)^{*}$ and the denominator is a certain 
Non-commutative formal deformations

ideal determined by $\operatorname{Ext}^{2}(F, F)^{*}$, an ideal generated by power series on a basis of $\operatorname{Ext}^{1}(F, F)^{*}$ corresponding to the members of a basis of $\operatorname{Ext}^{2}(F, F)^{*}$.

But it is more natural to consider the completed (non-symmetric) tensor algebra. We obtain the non-commutative $(N C)$ deformation algebra, the parameter algebra of a versal $N C$ deformation

$$
\hat{R}=k\left\langle\left\langle\operatorname{Ext}^{1}(F, F)^{*}\right\rangle\right\rangle /\left(\operatorname{Ext}^{2}(F, F)^{*}\right)
$$

where $k\left\langle\left\langle\operatorname{Ext}^{1}(F, F)^{*}\right\rangle\right\rangle$ is the completed tensor algebra

$$
\begin{aligned}
& \hat{T}_{k}^{\bullet} \operatorname{Ext}^{1}(F, F)^{*}=\prod_{i=0}^{\infty}\left(\operatorname{Ext}^{1}(F, F)^{*}\right)^{\otimes i} \\
& =k \times \operatorname{Ext}^{1}(F, F)^{*} \times\left(\operatorname{Ext}^{1}(F, F)^{*} \otimes \operatorname{Ext}^{1}(F, F)^{*}\right) \times \ldots
\end{aligned}
$$

and the denominator is a certain two sided ideal determined by $\operatorname{Ext}^{2}(F, F)^{*}$.

The abstract existence of a versal (formal) NC deformation is proved in the same way as in the case of commutative deformations ([11], [10]).

We can describe a versal deformation, as well as proving its existence, by using $A^{\infty}$-algebra formalism. Such a description is apparently well known to experts, e.g., [12] $\S 4$. But we use injective resolutions instead of locally free resolutions. This has advantage that our argument works not only for non-smooth non-projective varieties $X$ but also for objects in a $k$ linear abelian category with enough injectives. We also put emphasis on the non-commutativity of the parameter algebras. We treat only formal deformations, but there are results on the convergence (cf. Remarks 7.11 and 7.12).

The abstract description of the versal deformation using an $A^{\infty}$-algebra does not necessarily give solutions to explicit deformation problems because it involves injective resolutions etc. So we consider simple but non-trivial examples where the versal deformations are explicitly calculated. We prove that the versal deformation of a structure sheaf of a subvariety is described by a left ideal (Lemma 7.6). We apply this for lines in a projective space and prove that the relation ideal is generated by quadratic NC polynomials. We also calculate the relation $\mathrm{NC}$ polynomials for deformations of conics and prove that they have degree 3 .

The content of this paper is as follows. In $\S 2$, we give a definition of noncommutative deformations of a coherent sheaf, and express NC deformations as a change of differentials in an injective resolution. We describe them by using Maurer-Cartan equation in a differential graded associative algebra.

We review the theory of $A^{\infty}$-algebras in $\S 3$ in order to use it in later sections. In $\S 4$, we describe a versal deformation and the deformation algebra, the parameter algebra of a versal deformation, by using a minimal model $A^{\infty}$-algebra of the DG-algebra considered in $\S 2$. The advantage of 
Non-commutative formal deformations

the minimal model $A^{\infty}$ formulation is that the vector spaces are finite dimensional for fixed degrees, while the DG algebra is infinite dimensional in each degree. In order to achieve this, we need to introduce infinitely many multi-linear maps. We prove the versality of the deformation constructed by using the injective resolution (Theorem 4.6). We extend the whole theory to its refined version of multi-pointed NC deformations (Theorem 5.2) in $\S 5$. We make some remarks on the relationship of $\mathrm{NC}$ deformations and iterated self extensions in $\S 6$.

We consider some explicit non-trivial examples in $§ 7$. In Example 7.8 on lines in a projective space, we prove that the higher multiplications $m_{i}$ for $i \geq 3$ vanish, while in Example 7.9 on conics in $\mathbf{P}^{4}$, we prove that $m_{3} \neq 0$ but $m_{i}=0$ for $i \geq 4$.

The author would like to thank Professors Keiji Oguiso, Spela Spenko and Michel Van den Bergh for the information on the refernces [3] and [13] in Remark 7.10. The author would also like to thank Professors Yukinobu Toda and Zheng Hua for useful discussions (cf. Remarks 7.11 and 7.12).

\section{Non-COMmutative Deformations ANd DG ALGEBRA}

We consider 1-pointed non-commutative (NC) deformations of a coherent sheaf in this section. The extension to multi-pointed case is treated in a later section.

Let $X$ be an algebraic variety defined over a field $k$, and let $F$ be a coherent sheaf on $X$ with proper support.

Let $\left(\mathrm{Art}_{k}\right)$ be the category of associative $k$-algebras $R$ with a maximal two-sided ideal $M$ such that $R$ is a finite dimensional $k$-module, $R / M \cong k$, and that $M^{n+1}=0$ for some $n$. It follows that $R / M$ is the only simple $R$-module and any finitely generated $R$-module is obtained as a successive extension of $R / M$.

Definition 2.1. Let $X, F$ be as above, $R \in\left(\operatorname{Art}_{k}\right)$, let $F_{R}$ be a left $R \otimes_{k} \mathcal{O}_{X^{-}}$ module which is coherent as an $\mathcal{O}_{X}$-module, and let $\phi: R / M \otimes_{R} F_{R} \cong F$ be an isomorphism. Then a pair $\left(F_{R}, \phi\right)$ is said to be a non-commutative deformation of $F$ over $R$, if $F_{R}$ is flat as a left $R$-module.

Unless $M=0$ and $R=k$, we can define a two-sided ideal $J=M^{n}$ for the maximal integer $n$ such that $M^{n} \neq 0$. Then we have $M J=0$. If we put $R^{\prime}=R / J$, then we have $\operatorname{dim}_{k} R^{\prime}<\operatorname{dim}_{k} R$. We use this fact for the purpose of inductive argument on $\operatorname{dim}_{k} R$

We will describe NC deformation by using injective resolutions.

Lemma 2.2. Let $F$ be a coherent sheaf on $X$. Then there is an injective $O_{X}$-homomorphism $i: F \rightarrow I$ to an injective $O_{X}$-module which satisfies the following condition: for any deformations $F_{R}$ of $F$ over any $R \in\left(\right.$ Art $\left._{k}\right)$, there are injective $R \otimes_{k} O_{X}$-module homomorphisms $i_{R}: F_{R} \rightarrow R \otimes_{k} I$ such that $R / M \otimes_{R} i_{R}=i$. 
Non-commutative formal deformations

Proof. For any point $x \in X$, we define stalks of $I$ by $I_{x}=H_{0} m_{k}\left(O_{X, x}, F_{x}\right)$. Then $I_{x}$ has an $O_{X, x}$-module structure given by $a f(b)=f(a b)$ for $a, b \in O_{X, x}$ and $f \in I_{x}$. We claim that $I_{x}$ is an injective $O_{X, x}$-module. Indeed, for any $O_{X, x}$-module $M$, the map

$$
h: \operatorname{Hom}_{k}\left(M, F_{x}\right) \rightarrow \operatorname{Hom}_{O_{x}}\left(M, I_{x}\right)
$$

given by $h(f)(m)(a)=f(a m)$ for $f \in H_{0 m}\left(M, F_{x}\right), m \in M$ and $a \in$ $O_{X, x}$, is bijective with inverse given by $h^{-1}(g)(m)=g(m)(1)$ for $g \in$ $\operatorname{Hom}_{O_{x}}\left(M, I_{x}\right)$.

There is a natural injective $O_{X, x}$-homomorphism $i_{x}: F_{x} \rightarrow I_{x}$ defined by $i_{x}(c)(a)=a c$ for $c \in F_{x}$ and $a \in O_{X, x}$. We define an $O_{X}$-module $I$ by $I(U)=\prod_{x \in U} I_{x}$ for open subsets $U \subset X$. Then $I$ is an injective $O_{X}$-module with a natural injective $O_{X}$-homomorphism $i: F \rightarrow I$.

Since the stalk $F_{R, x}$ has an $R \otimes_{k} O_{X, x}$-module structure, the $k$-module $I_{R, x}=H_{o m}\left(O_{X, x}, F_{R, x}\right)$ has the induced $R \otimes_{k} O_{X, x}$-module structure given by $\operatorname{raf}(b)=r f(a b)$ for $a, b \in O_{X, x}, r \in R$ and $f \in I_{R, x}$. We define $I_{R}$ by $I_{R}(U)=\prod_{x \in U} I_{R, x}$. Then $I_{R}$ is again an injective module as an $O_{X}$-module, and there is a natural injective $R \otimes_{k} O_{X}$-homomorphism $i_{R}: F_{R} \rightarrow I_{R}$.

The natural surjective $O_{X}$-homomorphism $F_{R} \rightarrow F$ induces a surjective $R \otimes_{k} O_{X}$-homomorphism $I_{R} \rightarrow I$. Since $I_{R}$ is $O_{X}$-injective, there is a splitting $O_{X}$-homomorphism $I \rightarrow I_{R}$. By scalar extension, we obtain an $R \otimes_{k} O_{X}$-homomorphism $R \otimes_{k} I \rightarrow I_{R}$, which is bijective due to the flatness of $F_{R}$ over $R$. Therefore the lemma is proved.

The above lemma is non-trivial in some sense because $R \otimes_{k} I$ appears in the middle of the flow of arrows in the following diagram:

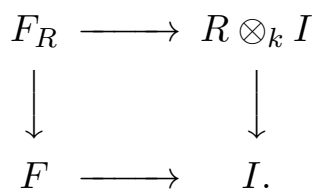

Corollary 2.3. There is an injective resolution

$$
0 \rightarrow F \rightarrow I^{0} \rightarrow I^{1} \rightarrow I^{2} \rightarrow \ldots
$$

as $O_{X}$-modules such that, for any deformation $F_{R}$ of $F$ over $R \in\left(\right.$ Art $\left._{k}\right)$, there is an exact sequence of $R \otimes_{k} O_{X}$-modules

$$
0 \rightarrow F_{R} \rightarrow R \otimes_{k} I^{0} \rightarrow R \otimes_{k} I^{1} \rightarrow R \otimes_{k} I^{2} \rightarrow \ldots
$$

which is reduced to the first exact sequence when the functor $R / M \otimes_{R}$ is applied.

Proof. We apply the lemma to the cokernels.

We describe NC deformations by using differential graded (DG) associative algebras. Let $F \rightarrow I^{\bullet}$ be an injective resolution as above, and let

$$
A=\operatorname{Hom}^{\bullet}\left(I^{\bullet}, I^{\bullet}\right)=\bigoplus_{i \in Z} \operatorname{Hom}^{i}\left(I^{\bullet}, I^{\bullet}\right)
$$


Non-commutative formal deformations

be the associative DG algebra of graded homomorphisms, where

$$
\operatorname{Hom}^{i}\left(I^{\bullet}, I^{\bullet}\right)=\prod_{m=0}^{\infty} \operatorname{Hom}\left(I^{m}, I^{m+i}\right)
$$

is the $i$-th graded piece, and the differential of $A$ is given by

$$
d_{A} f=d_{I} f-(-1)^{i} f d_{I}
$$

for $f \in \operatorname{Hom}^{i}\left(I^{\bullet}, I^{\bullet}\right)$, where $d_{I}$ denotes the differential of $I$.

Lemma 2.4. Let $(R, M) \in\left(\right.$ Art $\left._{k}\right)$, and let $y \in M \otimes A^{1}$. Let $d_{R, I}+y$ be an endomorphism of degree 1 of a graded $R \otimes_{k} \mathcal{O}_{X}$-module $R \otimes_{k} I^{\bullet}$, where $d_{R, I}=1_{R} \otimes d_{I}$ denotes the scalar extension of $d_{I}$. Then the following hold:

(1) $\left(d_{R, I}+y\right)^{2}=0$ if and only if the Maurer-Cartan equation

$$
d_{R, A} y+y^{2}=0
$$

is satisfied, where $d_{R, A}=1_{R} \otimes d_{A}$ is the scalar extension of $d_{A}$.

(2) In this case, $\mathcal{H}^{p}\left(R \otimes_{k} I^{\bullet}, d_{R, I}+y\right)=0$ for $p>0$ and $F_{R}:=\mathcal{H}^{0}\left(R \otimes_{k}\right.$ $\left.I^{\bullet}, d_{R, I}+y\right)$ is flat over $R$.

Proof. (1) We have $\left(d_{R, I}+y\right)\left(d_{R, I}+y\right)=d_{R, I} y+y d_{R, I}+y^{2}=d_{R, A} y+y^{2}$.

(2) We proceed by induction on $\operatorname{dim}_{k} R$. We take a two-sided ideal $J$ such that $M J=0$, and let $R^{\prime}=R / J$. Then we have an exact sequence of complexes

$$
0 \rightarrow J \otimes I^{\bullet} \rightarrow R \otimes I^{\bullet} \rightarrow R^{\prime} \otimes I^{\bullet} \rightarrow 0 .
$$

The associated long exact sequence yields the result.

The existence of a versal deformation, or a hull, for NC deformations is proved in the same way as in the case of commutative deformations ([11], [10]). One can describe a versal deformation using the formalism of $A^{\infty}$. algebras as explained in subsequent sections.

\section{REVIEW ON $A^{\infty}$-ALGEBRA}

We recall the definition of $A^{\infty}$-algebras (cf. [9]).

Definition 3.1. (1) Let $A=\bigoplus_{i \in \mathbf{Z}} A_{i}$ be a graded $k$-module. An $A^{\infty}$ algebra structure consists of $k$-linear maps $m_{i}: A^{\otimes i} \rightarrow A$ of degree $2-i$ for $i \geq 1$ satisfying the following relations:

$$
\sum_{r, t \geq 0, s \geq 1, r+s+t=i}(-1)^{r s+t} m_{r+1+t}\left(1^{\otimes r} \otimes m_{s} \otimes 1^{\otimes t}\right)=0 .
$$

For example,

$$
\begin{aligned}
& i=1: m_{1} m_{1}=0 \text {. } \\
& i=2: m_{1} m_{2}+m_{2}\left(-m_{1} \otimes 1-1 \otimes m_{1}\right)=0 . \\
& i=3: m_{1} m_{3}+m_{2}\left(-m_{2} \otimes 1+1 \otimes m_{2}\right) \\
& +m_{3}\left(m_{1} \otimes 1 \otimes 1+1 \otimes m_{1} \otimes 1+1 \otimes 1 \otimes m_{1}\right)=0 .
\end{aligned}
$$


Non-commutative formal deformations

(2) Let $A, B$ be $A^{\infty}$-algebras. An $A^{\infty}$-algebra homomorphism $f: A \rightarrow B$ consists of $k$-linear maps $f_{i}: A^{\otimes i} \rightarrow B$ of degree $1-i$ for $i \geq 1$ satisfying the following relations:

$$
\begin{aligned}
& \sum_{r, t \geq 0, s \geq 1, r+s+t=i}(-1)^{r s+t} f_{r+1+t}\left(1^{\otimes r} \otimes m_{s}^{A} \otimes 1^{\otimes t}\right) \\
= & \sum_{r \geq 1, i_{1}+\cdots+i_{r}=i}(-1)^{\sum_{1 \leq j<k \leq r} i_{j}\left(i_{k}+1\right)} m_{r}^{B}\left(f_{i_{1}} \otimes \cdots \otimes f_{i_{r}}\right) .
\end{aligned}
$$

For example,

$$
\begin{aligned}
i=1: & f_{1} m_{1}^{A}=m_{1}^{B} f_{1} . \\
i=2: & f_{1} m_{2}^{A}+f_{2}\left(-m_{1}^{A} \otimes 1-1 \otimes m_{1}^{A}\right)=m_{1}^{B} f_{2}+m_{2}^{B}\left(f_{1} \otimes f_{1}\right) . \\
i=3: & f_{1} m_{3}^{A}+f_{2}\left(-m_{2}^{A} \otimes 1+1 \otimes m_{2}^{A}\right) \\
& \quad+f_{3}\left(m_{1}^{A} \otimes 1 \otimes 1+1 \otimes m_{1}^{A} \otimes 1+1 \otimes 1 \otimes m_{1}^{A}\right) \\
& =m_{1}^{B} f_{3}+m_{2}^{B}\left(-f_{1} \otimes f_{2}+f_{2} \otimes f_{1}\right)+m_{3}^{B}\left(f_{1} \otimes f_{1} \otimes f_{1}\right) .
\end{aligned}
$$

A $D G$ (differential graded) associative algebra is a special case of an $A^{\infty}$ algebra where $m_{1}$ is the differential, $m_{2}$ is the associative algebra multiplication, and $m_{i}=0$ for $i \geq 3$.

Let $A$ be a DG algebra. Then its cohomology group $H(A)=\bigoplus_{i} H^{i}(A)$ is a graded $k$-module.

Theorem 3.2 (Kadeishvili [8]). Let $A$ be a DG associative algebra. Then there is an $A^{\infty}$-algebra structure on the cohomology group $H(A)$ such that $m_{1}=0, m_{2}$ is induced from the algebra multiplication $m_{2}^{A}$ of $A$, and that there is a morphism of $A^{\infty}$-algebras $f: H(A) \rightarrow A$ such that $f_{1}$ lifts the identity of $H(A)$.

sketch of proof. We define $k$-linear maps $m_{n}: H(A)^{\otimes n} \rightarrow H(A)$ of degree $2-n$ and $f_{n}: H(A)^{\otimes n} \rightarrow A$ of degree $1-n$ by induction on $n \geq 1$, which satisfy the following relations:

$$
\begin{aligned}
& \text { (1) } \sum_{r, t \geq 0, s \geq 2, r+s+t=n}(-1)^{r s+t} m_{r+1+t}\left(1^{\otimes r} \otimes m_{s} \otimes 1^{\otimes t}\right)=0 . \\
& \sum_{r, t \geq 0, s \geq 2, r+s+t=n}(-1)^{r s+t} f_{r+1+t}\left(1^{\otimes r} \otimes m_{s} \otimes 1^{\otimes t}\right) \\
& =m_{1}^{A} f_{n}+\sum_{i=1}^{n-1}(-1)^{i(n-i+1)} m_{2}^{A}\left(f_{i} \otimes f_{n-i}\right)
\end{aligned}
$$

where $m_{1}^{A}=d_{A}$ and $m_{2}^{A}$ is the associative multiplication.

First we set $m_{1}=0$. Let us choose $f_{1}: H(A) \rightarrow A$ to be any $k$-linear map which sends cohomology classes to their representatives. 
Non-commutative formal deformations

Now assume that $m_{i}$ and $f_{i}$ are already defined for $i<n$. Let $U_{n}$ : $H(A)^{\otimes n} \rightarrow A$ be a $k$-linear map of degree $2-n$ defined by

$$
\begin{aligned}
U_{n}= & \sum_{i=1}^{n-1}(-1)^{i(n-i+1)} m_{2}^{A}\left(f_{i} \otimes f_{n-i}\right) \\
& -\sum_{r, t \geq 0,2 \leq s \leq n-1, r+s+t=n}(-1)^{r s+t} f_{r+1+t}\left(1^{\otimes r} \otimes m_{s} \otimes 1^{\otimes t}\right) .
\end{aligned}
$$

For example, $U_{2}=m_{2}^{A}\left(f_{1} \otimes f_{1}\right)$. Then the condition (2) becomes

$$
m_{1}^{A} f_{n}+U_{n}=f_{1} m_{n} .
$$

A complicated calculation shows that $m_{1}^{A} U_{n}=0$, where we need to be careful on the sign changes.

We define $m_{n}=\left[U_{n}\right.$ ], where [ ] denotes the cohomology class in $H(A)$. Then it follows that $f_{1} m_{n}-U_{n} \in \operatorname{Im}\left(m_{1}^{A}\right)$. We choose any $k$-linear map $f_{n}$ such that $m_{1}^{A} f_{n}=f_{1} m_{n}-U_{n}$, then (2) is satisfied. Then we can check the relation (1) by a complicated calculation again.

The composition of $A^{\infty}$-morphisms $f: A \rightarrow B$ and $g: B \rightarrow C$ is defined as follows:

$$
(g \circ f)_{n}=\sum_{r \geq 1, \sum i_{j}=n}(-1)^{\sum_{j<k} i_{j}\left(i_{k}+1\right)} g_{r} \circ\left(f_{i_{1}} \otimes \cdots \otimes f_{i_{r}}\right)
$$

The identity morphism $f=1: A \rightarrow A$ is defined by $f_{1}=1$ and $f_{n}=0$ for $n \geq 2$.

Proposition 3.3. Let $A$ be a DG algebra, and let $f: H(A) \rightarrow A$ be the $A^{\infty}$ morphism obtained in the previous theorem. Then there is an $A^{\infty}$-algebra morphism $g: A \rightarrow H(A)$ such that $g \circ f=1_{H(A)}$.

Proof. We will define the $g_{n}$ inductively. The conditions are

$$
\begin{aligned}
& \sum_{r, t \geq 0, r+1+t=n}(-1)^{r+t} g_{r+1+t}\left(1^{\otimes r} \otimes m_{1}^{A} \otimes 1^{\otimes t}\right) \\
+ & \sum_{r, t \geq 0, r+2+t=n}(-1)^{t} g_{r+1+t}\left(1^{\otimes r} \otimes m_{2}^{A} \otimes 1^{\otimes t}\right) \\
= & \sum_{r \geq 2, \sum i_{j}=n}(-1)^{\sum_{j<k} i_{j}\left(i_{k}+1\right)} m_{r}^{H(A)}\left(g_{i_{1}} \otimes \cdots \otimes g_{i_{r}}\right),
\end{aligned}
$$

$g_{1} f_{1}=1$, and

$$
\sum_{r \geq 1, \sum i_{j}=n}(-1)^{\sum_{j<k} i_{j}\left(i_{k}+1\right)} g_{r} \circ\left(f_{i_{1}} \otimes f_{i_{r}}\right)=0
$$

for $n \geq 2$.

If the $g_{i}$ for $i<n$ are already determined, then $g_{n}$ is chosen such that it has given values on $f_{1}(A)^{\otimes n}$ and the $k$-subspace $V$ of $A^{\otimes n}$ generated by elements of the form $x_{1} \otimes \cdots \otimes x_{r} \otimes d x_{r+1} \otimes x_{r+2} \otimes \cdots \otimes x_{n}$. Such a $g_{n}$ exists because $f_{1}(A)^{\otimes n} \cap V=0$. 
Non-commutative formal deformations

\section{Description using $A^{\infty}$-Structure}

Let $F$ be a coherent sheaf on an algebraic variety $X$, and let $A=$ $\operatorname{Hom}^{\bullet}\left(I^{\bullet}, I^{\bullet}\right)$ be the DG algebra considered in $\S 2$. We know that $H^{p}(A)=$ $\operatorname{Ext}^{p}(F, F)$. The cohomology space $H(A)$ has an $A^{\infty}$-structure, and there are $A^{\infty}$-morphisms $f: H(A) \rightarrow A$ and $g: A \rightarrow H(A)$. We will describe versal NC deformation of $F$ using these $A^{\infty}$-algebras and morphisms.

In general, for $R \in\left(\operatorname{Art}_{k}\right)$, we define $m_{R, n}: R \otimes_{k} H^{1}(A)^{\otimes n} \rightarrow R \otimes_{k} H^{2}(A)$, $f_{R, n}: R \otimes_{k} H^{1}(A)^{\otimes n} \rightarrow R \otimes_{k} H^{1}(A)$ and so on by the extensions of scalars.

We consider the Maurer-Cartan equation in $A^{\infty}$-algebras using the following proposition:

Proposition 4.1. Let $(R, M) \in\left(\right.$ Art), and let $f: A \rightarrow B$ be an $A^{\infty}$ morphism. Let $x \in M \otimes A^{1}$ and $y=\sum_{i>1} f_{R, i}\left(x^{\otimes i}\right) \in M \otimes B^{1}$. If $x$ satisfies the Maurer-Cartan equation

$$
\sum_{i \geq 1} m_{R, i}^{A}\left(x^{\otimes i}\right)=0 \in R \otimes A^{2}
$$

then so does $y$ :

$$
\sum_{i \geq 1} m_{R, i}^{B}\left(y^{\otimes i}\right)=0 \in R \otimes B^{2} .
$$

We note that the sums are finite because $M$ is nilpotent.

Proof.

$$
\begin{aligned}
& \sum_{n \geq 1} m_{n}^{B}\left(y^{\otimes n}\right) \\
& =\sum_{n, i_{1}, \ldots, i_{n} \geq 1} m_{n}^{B}\left(f_{i_{1}}\left(x^{\otimes i_{1}}\right) \otimes \cdots \otimes f_{i_{n}}\left(x^{\otimes i_{n}}\right)\right) \\
& =\sum_{n, i_{1}, \ldots, i_{n} \geq 1}(-1)^{\sum_{j<k} i_{j}\left(1-i_{k}\right)} m_{n}^{B}\left(f_{i_{1}} \otimes \cdots \otimes f_{i_{n}}\right)\left(x^{\otimes\left(\sum_{j=1}^{n} i_{j}\right)}\right) \\
& =\sum_{r, t \geq 0, s \geq 1}(-1)^{r s+t} f_{r+1+t}\left(1^{\otimes r} \otimes m_{s}^{A} \otimes 1^{\otimes t}\right)\left(x^{\otimes(r+s+t)}\right) \\
& =\sum_{r, t \geq 0, s \geq 1}(-1)^{t} f_{r+1+t}\left(x^{\otimes r} \otimes m_{s}^{A}\left(x^{\otimes s}\right) \otimes x^{\otimes t}\right) \\
& =0
\end{aligned}
$$

where we dropped the subscripts $R$ for simplicity.

In the above argument, we followed the Koszul rule of the signs:

$$
(x \otimes y)(z \otimes w)=(-1)^{\operatorname{deg}(y) \operatorname{deg}(z)} x z \otimes y w .
$$

Lemma 4.2. Let $A$ be a $D G$ algebra, and let $f: H(A) \rightarrow A$ and $g: A \rightarrow$ $H(A)$ be $A^{\infty}$-morphisms such that $g \circ f=1_{H(A)}$. Let $(R, M) \in\left(\right.$ Art $\left._{k}\right)$, let $x \in M \otimes H^{1}(A)$, and let $y=\sum_{i \geq 1} f_{R, i}\left(x^{\otimes i}\right) \in M \otimes A^{1}$. Then $x=$ $\sum_{i \geq 1} g_{R, i}\left(y^{\otimes i}\right)$. 
Non-commutative formal deformations

Proof. We have

$$
\begin{aligned}
& \sum_{n \geq 1} g_{n}\left(y^{\otimes n}\right) \\
& =\sum_{n, i_{1}, \ldots, i_{n} \geq 1} g_{n}\left(f_{i_{1}}\left(x^{\otimes i_{1}}\right) \otimes \cdots \otimes f_{i_{n}}\left(x^{\otimes i_{n}}\right)\right) \\
& =\sum_{n, i_{1}, \ldots, i_{n} \geq 1}(-1)^{\sum_{j<k} i_{j}\left(1-i_{k}\right)} g_{n}\left(f_{i_{1}} \otimes \cdots \otimes f_{i_{n}}\right)\left(x^{\otimes\left(\sum_{j=1}^{n} i_{j}\right)}\right) \\
& =\sum_{n \geq 1}(g \circ f)_{n}\left(x^{\otimes n}\right) \\
& =x
\end{aligned}
$$

Corollary 4.3. $x \in M \otimes H^{1}(A)$ satisfies the $M C$ equation if and only if $y=\sum_{i \geq 1} f_{R, i}\left(x^{\otimes i}\right) \in M \otimes A^{1}$ satisfies the $M C$ equation.

Now we construct a versal deformation over its parameter algebra, called the deformation ring. Let $\left\{v_{i}\right\}$ be a basis of $H^{1}(A)$. Let $x \in H^{1}(A)^{*} \otimes H^{1}(A)$ be the tautological element corresponding to the identity of $H^{1}(A)$. Then we can write

$$
x=\sum_{i} v_{i}^{*} \otimes v_{i}
$$

for the dual basis $\left\{v_{i}^{*}\right\}$ of $H^{1}(A)^{*}$.

Let $m_{n}: T^{n} H^{1}(A)=\left(H^{1}(A)\right)^{\otimes n} \rightarrow H^{2}(A)$ be the $A^{\infty}$-multiplication, let $m_{[2, n]}=\sum_{i=2}^{n} m_{i}: \bigoplus_{i=0}^{n} T^{i} H^{1}(A) \rightarrow H^{2}(A)$, and let $m_{[2, n]}^{*}: H^{2}(A)^{*} \rightarrow$ $\bigoplus_{i=0}^{n} T^{i} H^{1}(A)^{*}$ be the dual map. We define

$$
R_{n}=\bigoplus_{i=0}^{n} T^{i} H^{1}(A)^{*} / m_{[2, n]}^{*} H^{2}(A)^{*}
$$

where $R_{0}=k, R_{1}=k \oplus H^{1}(A)^{*}$, and the product of total degree more than $n$ is set to be zero. There are natural surjective ring homomorphisms $R_{n} \rightarrow R_{n^{\prime}}$ for $n>n^{\prime}$. Let $M_{n}=\operatorname{Ker}\left(R_{n} \rightarrow R_{0}=k\right)$. Then we have $M_{n}^{n+1}=0$. We define the formal completion

$$
\hat{R}=\lim _{\longleftarrow} R_{n}=\hat{T}^{\bullet} H^{1}(A)^{*} / m^{*} H^{2}(A)^{*}
$$

where we set formally $m=\sum_{i=2}^{\infty} m_{i}$.

Let $m_{R_{n}, i}: R_{n} \otimes T^{i} H^{1}(A) \rightarrow R_{n} \otimes H^{2}(A)$ be the map obtained from $m_{n}$ by scalar extension.

Lemma 4.4. $R_{n}$ is the largest quotient ring of $\bigoplus_{i=0}^{n} T^{i} H^{1}(A)^{*}$ such that $\sum_{i=2}^{n} m_{R_{n}, i}\left(x^{\otimes i}\right)=0$ in $R_{n} \otimes H^{2}(A)$. 
Non-commutative formal deformations

Proof. Let $\left\{w_{j}\right\}$ be a basis of $H^{2}(A)$, and let $\left\{w_{i}^{*}\right\}$ be the dual basis of $H^{2}(A)^{*}$. We write $m_{k}\left(v_{i_{1}} \otimes \cdots \otimes v_{i_{k}}\right)=\sum_{j} a_{i_{1}, \ldots, i_{k}, j} w_{j}$. Then

$$
\sum_{k=2}^{n} m_{k}^{*}\left(w_{j}^{*}\right)=\sum_{k, i_{1}, \ldots, i_{k}} a_{i_{1}, \ldots, i_{k}, j} v_{i_{1}}^{*} \otimes \cdots \otimes v_{i_{k}}^{*} .
$$

We have

$$
\begin{aligned}
& \sum_{k=2}^{n} m_{R, k}\left(x^{\otimes k}\right)=\sum_{k, i_{1}, \ldots, i_{k}} m_{R, k}\left(v_{i_{1}}^{*} \otimes \cdots \otimes v_{i_{k}}^{*} \otimes v_{i_{1}} \otimes \cdots \otimes v_{i_{k}}\right) \\
& =\sum_{k, i_{1}, \ldots, i_{k}, j} a_{i_{1}, \ldots, i_{k}, j} v_{i_{1}}^{*} \otimes \cdots \otimes v_{i_{k}}^{*} \otimes w_{j} .
\end{aligned}
$$

Therefore $\sum_{k=2}^{n} m_{R, k}\left(x^{\otimes k}\right)=0$ in $R \otimes H^{2}(A)$ if and only if $\sum_{k=2}^{n} m_{k}^{*}\left(w_{j}^{*}\right)=0$ in $R$ for all $j$.

Corollary 4.5. Let $y_{n}=\sum_{i=1}^{n} f_{R_{n}, i}\left(x^{\otimes i}\right) \in R_{n} \otimes A^{1}$. Then $\left(d_{R_{n}, I}+y_{n}\right)^{2}=0$ as an endomorphism of $R_{n} \otimes I^{*}$, where we denote $d_{R_{n}, I}=1_{R_{n}} \otimes d_{I}$.

By Lemma 2.4, we define an NC deformation

$$
F_{n}=\operatorname{Ker}\left(d_{R_{n}, I}+y_{n}: R_{n} \otimes I^{0} \rightarrow R_{n} \otimes I^{1}\right)
$$

of $F$ over $R_{n}$.

The following theorem is apparently well-known to experts (cf. [12]):

Theorem 4.6. Let $\hat{F}=\lim F_{n}$ be the inverse limit. Then the formal deformation $\hat{F}$ over $\hat{R}$ is a versal non-commutative deformation of $F$

Proof. We have to prove the following statement: "Let $R$ be a quotient algebra of $\bigoplus_{i=0}^{n} T^{i} H^{1}(A)^{*}$ such that $R_{n}$ is its quotient algebra. Assume that there is an element $y \in R \otimes A^{1}$ which satisfies the Maurer-Cartan equation and induces $y_{n}$ on $R_{n} \otimes A^{1}$. Then $R=R_{n}$ ".

We will derive a contradiction assuming that $R \neq R_{n}$. We may assume that the images of $R$ and $R_{n}$ to quotient algebras of $\bigoplus_{i=0}^{n-1} T^{i} H^{1}(A)^{*}$ coincide. Let $y_{R}=\sum_{i=1}^{n} f_{R, i}\left(x^{\otimes i}\right) \in R \otimes A^{1}$ for $x=\sum v_{i}^{*} \otimes v_{i} \in H^{1}(A)^{*} \otimes H^{1}(A)$. We set $z=y-y_{R} \in M^{n} \otimes A^{1}$. We note that $y$ satisfies the MC equation over $R$ but $y_{R}$ does not, because neither does $x$ over $R$.

We have $y^{\otimes i}=\left(y_{R}+z\right)^{\otimes i}=y_{R}^{\otimes i}$ for $i \geq 2$, and we have $x=\sum_{i=1}^{n} g_{R, i}\left(y_{R}^{\otimes i}\right)$. Let $x^{\prime}=\sum_{i=1}^{n} g_{R, i}\left(y^{\otimes i}\right)$. Then we have $x^{\prime}=x+g_{R, 1}(z)$. It follows that $x^{\otimes i}=x^{\prime \otimes i}$ for $i \geq 2$. Since $y$ satisfies the MC equation over $R$, so does $x^{\prime}$. But since $m_{1}^{H(A)}=0$, we deduce that $x$ satisfies the MC equation over $R$, a contradiction.

The parameter algebra $\hat{R}$ of the versal deformation is called a deformation algebra of $F$. 
Non-commutative formal deformations

\section{1-POINTED VERSUS $r$-POINTED DEFORMATIONS}

Now we consider $r$-pointed deformations for a positive integer $r \geq 1$. If $r=1$, then they are $\mathrm{NC}$ deformations in the previous sections. It is a refined version in the case where the coherent sheaf $F$ has a direct sum decomposition to $r$ ordered factors $F=\bigoplus_{i=1}^{r} F_{i}$.

We consider the base ring $k^{r}$, the product ring of $r$ copies of $k$, instead of $k$. $F$ has a structure of a left $k^{r}$-module, where the orthogonal idempotents $e_{i}(1 \leq i \leq r)$ of $k^{r}$ correspond to the projections $F \rightarrow F_{i}=e_{i} F$.

Let $\left(A r t_{k}^{r}\right)$ be the category of pairs $(R, M)$ such that $R$ is an associative $k^{r}$ algebra with an augmentation $R \rightarrow k^{r}$ and $M$ is a two-sided ideal satisfying the conditions that $R$ is a finite dimensional $k$-module, $R / M \cong k^{r}$, and that $M^{n+1}=0$ for some $n$. We have $R / M \cong \bigoplus_{i=1}^{r} R / M_{i}$ for maximal two-sided ideals $M_{i}$. It follows that the $R / M_{i}$ are the only simple $R$-modules and any finitely generated $R$-module is obtained as a successive extension of the $R / M_{i}$ (cf. [6]).

Definition 5.1. Let $F=\bigoplus_{i=1}^{r} F_{i}$ be a direct sum of coherent sheaves with proper supports on an algebraic variety $X$ and $R \in\left(A r t_{k}^{r}\right)$. Let $F_{R}$ be a left $R \otimes_{k} \mathcal{O}_{X}$-module which is coherent as an $\mathcal{O}_{X}$-module. Then a pair $\left(F_{R}, \phi\right)$ is said to be an $r$-pointed non-commutative deformation of $F$ over $R$, if $F_{R}$ is flat as a left $R$-module and $\phi: k^{r} \otimes_{R} F_{R} \rightarrow F$ is an isomorphism.

The injective resolution $F \rightarrow I^{\bullet}$ are $k^{r}$-equivariant in the sense that $I^{\bullet}=\bigoplus_{i=1}^{r} I_{i}^{\bullet}$, where the $F_{i} \rightarrow I_{i}^{\bullet}$ are injective resolutions. The graded ring $A=\operatorname{Hom}^{\bullet}\left(I^{\bullet}, I^{\bullet}\right)$ has a structure of $k^{r}$-bimodules; we have a direct sum decomposition $\operatorname{Hom}^{\bullet}\left(I^{\bullet}, I^{\bullet}\right)=\bigoplus_{i, j=1}^{r} \operatorname{Hom}^{\bullet}\left(I_{i}^{\bullet}, I_{j}^{\bullet}\right)$. It is convenient to write $A$ in a matrix form $A_{i j}=\operatorname{Hom}^{\bullet}\left(I_{i}^{\bullet}, I_{j}^{\bullet}\right)$, because we have $A_{i j} A_{k l}=0$ if $j \neq k$.

The constructions of the deformation ring and the versal deformation are generalized from the 1-pointed case to the $r$-pointed case in the following way. The cohomology groups $H^{p}(A)=\operatorname{Ext}^{p}(F, F)$ have also $k^{r}$-bimodule structures. $H(A)=\bigoplus_{i} H^{i}(A)$ has an $A^{\infty}$-structure with a $k^{r}$-bimodule structure. If $n>0$, then there is an injective homomorphism from a direct summand

$$
T_{k^{r}}^{n} H^{1}(A) \rightarrow T_{k}^{n} H^{1}(A)
$$

between tensor products. For example,

$$
T_{k^{r}}^{2} H^{1}(A)=\bigoplus_{i, j, k} H^{1}(A)_{i j} \otimes H^{1}(A)_{j k} \subset T_{k}^{2} H^{1}(A) .
$$

The $A^{\infty}$-multiplications

$$
m_{n}^{r}: T_{k^{r}}^{n} H^{1}(A) \rightarrow H^{2}(A)
$$

for $n \geq 2$ are induced from the 1-pointed case $m_{n}=m_{n}^{1}$. We define

$$
R_{n}^{r}=\bigoplus_{i=0}^{n} T_{k^{r}}^{i} H^{1}(A)^{*} /\left(m_{[2, n]}^{r}\right)^{*} H^{2}(A)^{*}
$$


Non-commutative formal deformations

for $m_{[2, n]}^{r}=\sum_{i=2}^{n} m_{i}^{r}$, and

$$
\hat{R}^{r}=\lim _{\longleftarrow} R_{n}^{r}
$$

as before.

In order to define $\hat{F}^{r}$, we take the tautological element

$$
x=\sum_{i} v_{i}^{*} \otimes v_{i} \in H^{1}(A)^{*} \otimes_{k^{r}} H^{1}(A)
$$

again, where each $v_{i}$ belongs to a $H^{1}(A)_{s t}$ for some $s, t$ so that $v_{i}^{*}$ belongs to $\left(H^{1}(A)^{*}\right)_{t s}$. Let

$$
y_{n}^{r}=\sum_{i=1}^{n} f_{R_{n}^{r}, i}^{r}\left(x^{\otimes i}\right) \in R_{n}^{r} \otimes A^{1}
$$

where $f_{R_{n}^{r}, i}^{r}$ is induced from $f_{i}$. Then we define

$$
F_{n}^{r}=\operatorname{Ker}\left(d_{R_{n}^{r}, I}+y_{n}: R_{n}^{r} \otimes_{k^{r}} I^{0} \rightarrow R_{n}^{r} \otimes_{k^{r}} I^{1}\right)
$$

and

$$
\hat{F}^{r}=\lim _{\longleftarrow} F_{n}^{r} .
$$

We compare deformation rings

$$
\hat{R}^{1}=\lim _{\longleftarrow} R_{n}^{1}, \quad \hat{R}^{r}=\lim _{\longleftarrow} R_{n}^{r}
$$

of 1-pointed and $r$-pointed deformations. Their Zariski cotangent spaces are the same $H^{1}(A)^{*}=\left(\operatorname{Ext}^{1}(F, F)\right)^{*}$. The truncated deformation ring $R_{n}^{r}$ of $r$-pointed deformations is a quotient algebra of the tensor algebra over $k^{r}$ :

$$
\begin{aligned}
& T_{k^{r}}^{\bullet} H^{1}(A)^{*}=\prod_{i=0}^{\infty} T_{k^{r}}^{i} H^{1}(A)^{*} \\
& =k^{r} \times H^{1}(A)^{*} \times\left(H^{1}(A)^{*} \otimes_{k^{r}} H^{1}(A)^{*}\right) \times \ldots
\end{aligned}
$$

where the tensor products are taken over the base ring $k^{r}$.

There is a split surjective ring homomorphism $k^{r} \times T_{k}^{\bullet} H^{1}(A)^{*} \rightarrow T_{k^{r}}^{\bullet} H^{1}(A)^{*}$. We have

$$
T_{k^{r}}^{\bullet} H^{1}(A)^{*}=\left(k^{r} \times T_{k}^{\bullet} H^{1}(A)^{*}\right) / J
$$

where the ideal $J$ is generated by relations $\sum_{i=1}^{r} e_{i}=1$ and $H^{1}(A)_{i j}^{*} H^{1}(A)_{k l}^{*}=$ 0 for $j \neq k$. The degree 0 part of $T_{k^{r}}^{\bullet} H^{1}(A)^{*}$ is $k^{r}$, which is larger than $k$, but positive degree parts are quotients of the usual tensor products $T_{k}^{i} H^{1}(A)^{*}$. Therefore the $r$-pointed deformation ring $\hat{R}^{r}$ is not exactly a quotient of the 1-pointed deformation ring $\hat{R}^{1}$, but almost is. In particular, $r$-pointed deformations are derived from a special case of 1-pointed deformations.

We note that the deformation $F_{R_{n}^{r}}$ over $R_{n}^{r}$ is different from the one induced from the deformation $F_{R_{n}^{1}}$ by the natural ring homomorphism $R_{n}^{1} \rightarrow$ $R_{n}^{r}$. For example, $F_{R_{n}^{r}}$ is flat over $R_{n}^{r}$ and $k^{r} \otimes_{R_{n}^{r}} F_{R_{n}^{r}}=F$, but $k \otimes_{R_{n}^{1}} F_{R_{n}^{1}}=$ $F$. We have

$$
F_{R_{n}^{r}}=\left(R_{n}^{r} \otimes_{R_{n}^{1}} F_{R_{n}^{1}}\right) / N
$$


Non-commutative formal deformations

where $N$ is a submodule consisting of irrelevant factors of $F_{R_{n}^{1}}$ that are attached in the extension process; we have to attach $F_{i}$ 's instead of $F$ (cf. Example 7.3).

The following theorem is a consequence of Theorem 4.6:

Theorem 5.2. The formal deformation $\hat{F}^{r}$ of $F$ over $\hat{R}^{r}$ is a versal $r$ pointed non-commutative deformation of $F$ in the following sense. If $\left(F_{R}^{r}, \phi_{0}^{r}\right)$ is any $r$-pointed non-commutative deformation over $(R, M) \in\left(\right.$ Art $\left._{k}^{r}\right)$ such that $\phi_{0}^{r}: R / M \otimes_{k^{r}} F_{R} \rightarrow F$ is an isomorphism, then there exist an integer $n$ and a $k^{r}$-algebra homomorphism $\psi^{r}: R_{n}^{r} \rightarrow R$ such that there is an isomorphism $\phi^{r}: R \otimes_{R_{n}^{r}} F_{n}^{r} \rightarrow F_{R}$ which induces $\phi_{0}^{r}$ over $R / M$.

\section{REMARK ON UNIVERSAL EXTENSIONS}

We consider iterated self extensions of $F=\bigoplus_{i=1}^{r} F_{i}$ in this section. NC deformations of $F$ are iterated self extensions of $F$. Conversely, any iterated self extensions of $F$ are expected to be expressed as NC deformations of $F$, and the versal deformation is given by a tower of universal extensions. Indeed if $F$ is a simple collection, i.e., if $\operatorname{End}(F) \cong k^{r}$, then it is the case (6] Theorem 4.8). The point is that the parameter algebra in this case is naturally given as the endomorphism ring of the iterated non-trivial self extensions.

We define inductively a tower of universal extensions by $E_{0}^{r}=F$ and

$$
0 \rightarrow \operatorname{Ext}^{1}\left(E_{n}^{r}, F\right)^{*} \otimes_{k^{r}} F \rightarrow E_{n+1}^{r} \rightarrow E_{n}^{r} \rightarrow 0
$$

for $n \geq 0$, or equivalently

$$
0 \rightarrow \bigoplus_{j} \operatorname{Ext}^{1}\left(E_{n, i}^{r}, F_{j}\right)^{*} \otimes_{k} F_{j} \rightarrow E_{n+1, i}^{r} \rightarrow E_{n, i}^{r} \rightarrow 0
$$

where we note that $E_{n}^{r}=\bigoplus_{i=1}^{r} E_{n, i}^{r}$ is a left $k^{r}$-module and $\operatorname{Ext}^{1}\left(E_{n}^{r}, F\right)$ is a $k^{r}$-bimodule. The above exact sequence corresponds to a natural morphism

$$
\begin{aligned}
& \operatorname{Ext}^{1}\left(E_{n}^{r}, F\right)[-1] \otimes_{k^{r}} E_{n}^{r}=\bigoplus_{i, j} \operatorname{Ext}^{1}\left(E_{n, i}^{r}, F_{j}\right)[-1] \otimes_{k} E_{n, i}^{r} \\
& \rightarrow F=\bigoplus_{j} F_{j}
\end{aligned}
$$

in the derived category.

On the other hand, in the notation of the previous sections, from an exact sequence

$$
0 \rightarrow\left(M_{n+1}^{r}\right)^{n+1} \rightarrow R_{n+1}^{r} \rightarrow R_{n}^{r} \rightarrow 0
$$

we obtain an exact sequence

$$
0 \rightarrow\left(M_{n+1}^{r}\right)^{n+1} \otimes_{k^{r}} F \rightarrow F_{n+1}^{r} \rightarrow F_{n}^{r} \rightarrow 0
$$

where we note that $\left(M_{n+1}^{r}\right)^{n+1} \otimes_{R_{n+1}^{r}} F \cong\left(M_{n+1}^{r}\right)^{n+1} \otimes_{k^{r}} F$. 
Non-commutative formal deformations

We expect that (6.1) and (6.2) are isomorphic as exact sequences of $\mathcal{O}_{X^{-}}$ modules. For example, we have $M_{1} \cong M_{1}^{r} \cong \operatorname{Ext}^{1}(F, F)^{*}$, and this is the case for $n=0$.

In the case $n=1$, from an exact sequence

$$
\begin{aligned}
& M_{1}^{*} \otimes \operatorname{Hom}(F, F) \rightarrow \operatorname{Ext}^{1}(F, F) \rightarrow \operatorname{Ext}^{1}\left(F_{1}, F\right) \\
& \rightarrow M_{1}^{*} \otimes \operatorname{Ext}^{1}(F, F) \rightarrow \operatorname{Ext}^{2}(F, F)
\end{aligned}
$$

we deduce that

$$
\operatorname{Ext}^{1}\left(F_{1}, F\right)=\operatorname{Ker}\left(M_{1}^{*} \otimes \operatorname{Ext}^{1}(F, F) \rightarrow \operatorname{Ext}^{2}(F, F)\right) .
$$

Thus

$$
\operatorname{Ext}^{1}\left(F_{1}, F\right)^{*}=\operatorname{Coker}\left(m_{2}: \operatorname{Ext}^{2}(F, F)^{*} \rightarrow\left(\operatorname{Ext}^{1}(F, F)^{*}\right)^{\otimes 2}\right)=M_{2}^{2} .
$$

Thus each corresponding terms in (6.1) and (6.2) coincide for $n=1$.

We compare universal extensions corresponding to 1-pointed and $r$-pointed deformations:

Lemma 6.1. There are natural split surjective homomorphisms $E_{n}^{1} \rightarrow E_{n}^{r}$.

Proof. For $n=0$, we have $E_{0}^{1}=E_{0}^{r}=F$.

Assume that there is a split surjective homomorphism $E_{n}^{1} \rightarrow E_{n}^{r}$. Then there is an induced split surjective homomorphism

$$
\operatorname{Ext}^{1}\left(E_{n}^{1}, F\right)^{*} \rightarrow \operatorname{Ext}^{1}\left(E_{n}^{r}, F\right)^{*} .
$$

Since

$$
\begin{aligned}
& \operatorname{Ext}^{1}\left(E_{n}^{r}, F\right)^{*} \otimes_{k} F=\sum_{i, j} \operatorname{Ext}^{1}\left(E_{n}^{r}, F_{i}\right)^{*} \otimes_{k} F_{j} \\
& \operatorname{Ext}^{1}\left(E_{n}^{r}, F\right)^{*} \otimes_{k^{r}} F=\sum_{i} \operatorname{Ext}^{1}\left(E_{n}^{r}, F_{i}\right)^{*} \otimes_{k} F_{i}
\end{aligned}
$$

we have a split surjective homomorphism

$$
\operatorname{Ext}^{1}\left(E_{n}^{r}, F\right)^{*} \otimes_{k} F \rightarrow \operatorname{Ext}^{1}\left(E_{n}^{r}, F\right)^{*} \otimes_{k^{r}} F
$$

hence there is also a split surjective homomorphism $E_{n+1}^{1} \rightarrow E_{n+1}^{r}$.

\section{EXAMPLES}

We consider some examples of versal $\mathrm{NC}$ deformations in this section. We start with a trivial example:

Example 7.1. Let $F=\mathcal{O}_{x}$ be the structure sheaf of a point $x \in X$. We claim that the versal deformation $\hat{F}$ of $F$ is isomorphic to the deformation algebra $\hat{R}$, which is commutative and isomorphic to the formal completion of the local ring $\hat{O}_{X, x}$.

$F$ is a simple collection with $r=1$, i.e., a simple sheaf in this case, hence the versal deformation is given by the tower of universal extensions ([6] Theorem 4.8). Therefore it is sufficient to prove that any NC deformation $F_{R}$ of 
Non-commutative formal deformations

$F$ over some $R \in\left(\operatorname{Art}_{k}\right)$ obtained by successive non-trivial extensions is of the form $F_{R} \cong \mathcal{O}_{X} / J$ for an ideal $J$ such that $\operatorname{Supp}\left(F_{R}\right)=\{x\}$. We proceed by induction on $\operatorname{dim} R$. Let

$$
0 \rightarrow \mathcal{O}_{x} \rightarrow E \rightarrow \mathcal{O}_{X} / J \rightarrow 0
$$

be a non-trivial extension. Since $\operatorname{Ext}^{1}\left(\mathcal{O}_{X}, \mathcal{O}_{x}\right)=0$, the natural surjective homomorphism $\mathcal{O}_{X} \rightarrow \mathcal{O}_{X} / J$ lifts to a homomorphism $\mathcal{O}_{X} \rightarrow E$. Let $J^{\prime}$ be the kernel. Then there are homomorphism $\mathcal{O}_{X} / J^{\prime} \rightarrow E \rightarrow \mathcal{O}_{X} / J$ whose combination is surjective and the first homomorphism is injective. There are two cases: length $\left(\mathcal{O}_{X} / J^{\prime}\right)-\operatorname{length}\left(\mathcal{O}_{X} / J\right)=0$ or 1 . In the first case, we have $J=J^{\prime}$ and the homomorphism $E \rightarrow \mathcal{O}_{X} / J$ splits, a contradiction to the hypothesis that the extension is non-trivial. In the second case, we have $E=\mathcal{O}_{X} / J^{\prime}$, and the claim is proved after taking the inverse limit.

Remark 7.2. Let $F=\mathcal{O}_{x}$ for a smooth point $x \in X$. Then we have $\operatorname{Ext}^{p}\left(\mathcal{O}_{x}, \mathcal{O}_{x}\right) \cong \wedge^{p} k^{n}$ for $n=\operatorname{dim} X$. The deformation algebra is isomorphic to the formal power series ring $k\left[\left[x_{1}, \ldots, x_{n}\right]\right]$. There are no obstructions for commutative deformations of $F$, but the non-commutative deformations are highly obstructed.

Example 7.3. Let $X=\{x y=0\} \subset \mathbf{P}^{2}$ be the union of two distinct lines, and let $F=\mathcal{O}_{X} /(x) \oplus \mathcal{O}_{X} /(y):=F_{x} \oplus F_{y}$ be the sum of structure sheaves of these lines. We compare 1-pointed and 2-pointed deformations of $F$.

The 2-pointed deformation ring is calculated in [6] Example 5.5:

$$
\hat{R}^{2}=\left(\begin{array}{cc}
k & k t \\
k t & k
\end{array}\right) \quad \bmod \left(t^{2}\right)
$$

and $\operatorname{dim} \hat{R}^{2}=4$. On the other hand, the 1-pointed deformation ring is:

$$
\hat{R}^{1}=k[[x, y]] /(x y)=k\langle\langle x, y\rangle\rangle /(x y, y x)
$$

and $\operatorname{dim} \hat{R}^{1}=\infty$.

The corresponding deformations are as follows. There are non-trivial extensions

$$
\begin{aligned}
& 0 \rightarrow \mathcal{O}_{X} /(y) \rightarrow F_{1, x}^{2} \rightarrow \mathcal{O}_{X} /(x) \rightarrow 0 \\
& 0 \rightarrow \mathcal{O}_{X} /(x) \rightarrow F_{1, y}^{2} \rightarrow \mathcal{O}_{X} /(y) \rightarrow 0
\end{aligned}
$$

where $F_{1, x}^{2}$ (resp. $\left.F_{1, y}^{2}\right)$ are invertible sheaves on $X$ whose degrees on the irreducible components $L_{x}=\{y=0\}$ and $L_{y}=\{x=0\}$ of $X$ are $(0,1)$ (resp. $(1,0)$ ). We have

$$
\hat{F}^{2}=F_{1}^{2}=F_{1, x}^{2} \oplus F_{1, y}^{2} .
$$

On the other hand, we have

$$
F_{n}^{1} \cong\left(F_{1, x}^{2}\right)^{\oplus n} \oplus \mathcal{O}_{X} /(x) \oplus\left(F_{1, y}^{2}\right)^{\oplus n} \oplus \mathcal{O}_{X} /(y) .
$$

Example 7.4. Let $X=\left\{x^{2}+y^{2}+y^{3}=0\right\} \subset \mathbf{P}^{2}$ be a rational curve with one node, and let $F$ be the structure sheaf of the normalization of $X$. 
Non-commutative formal deformations

$X$ has a singularity which is analytically isomorphic to the singularity of the variety considered in the previous example. We have again $\hat{R}=$ $k[[x, y]] /(x y)$, and $\hat{F}$ becomes an invertible sheaf on an infinite chain of rational curves.

We have $\operatorname{End}_{X}(F)=k$ but $\operatorname{End}_{D_{s g}}(F)=k[t] /\left(t^{2}+1\right)([7])$.

Example 7.5 ([6] Example 5.8). Let $X=\mathbf{P}(1,2,3)$ be a weighted projective plane, and let $F=\mathcal{O}_{X}(1)$ be a reflexive sheaf of rank 1 corresponding to a line on $X$ connecting its two singular points.

Then the deformation ring for $\mathrm{NC}$ deformations is an infinite dimensional algebra $\hat{R}=k\langle\langle x, y\rangle\rangle /\left(x^{2}, y^{3}\right)$, where the generators $x, y$ respectively correspond to local extensions of $F$ near the singularities of types $\frac{1}{2}(1,1)$ and $\frac{1}{3}(1,2)$. But $\hat{R}^{a b}=k[[x]] /\left(t^{6}\right)$ is finite dimensional for commutative deformations.

Lemma 7.6. Let $X$ be a proper variety and let $Y$ be a closed subvariety. Let $F=\mathcal{O}_{Y}=\mathcal{O}_{X} / J$ be the structure sheaf of $Y$ regarded as an $\mathcal{O}_{X}$-module. Assume that $H^{0}\left(X, \mathcal{O}_{X}\right) \cong H^{0}\left(X, \mathcal{O}_{Y}\right) \cong k$ and $H^{1}\left(X, \mathcal{O}_{Y}\right)=0$. Then the versal deformation $\hat{F}$ of $F$ is of the form

$$
\hat{F}=\lim _{\longleftarrow}\left(R_{n} \otimes \mathcal{O}_{X}\right) / J_{n}
$$

for left $R_{n} \otimes \mathcal{O}_{X}$-ideals $J_{n}$. Moreover if $J \otimes \mathcal{O}_{X}(1)$ is generated by global sections $h_{i}(i=1, \ldots, r)$ for a very ample invertible sheaf $\mathcal{O}_{X}(1)$ on $X$ and if $H^{1}\left(X, J \otimes \mathcal{O}_{X}(1)\right)=0$, then there are global sections $\hat{h}_{i}$ of $\lim _{(}\left(J_{n} \otimes \mathcal{O}_{X}(1)\right)$ which generate $\lim _{\longleftarrow}\left(J_{n} \otimes \mathcal{O}_{X}(1)\right)$ and induce $h_{i} \in J \otimes \mathcal{O}_{X}(1)$.

Proof. Since $F$ is a simple sheaf, a versal NC deformation is obtained by a sequence of universal extensions. We prove that a deformation $F_{R}$ over $R \in\left(\operatorname{Art}_{k}\right)$ is of the form $\left(R \otimes \mathcal{O}_{X}\right) / J_{R}$ for a left $R \otimes \mathcal{O}_{X}$-ideal $J_{R}$ by induction on $\operatorname{dim}_{k} R=i$. Let

$$
0 \rightarrow \mathcal{O}_{Y} \rightarrow E \rightarrow\left(R^{\prime} \otimes \mathcal{O}_{X}\right) / J_{R^{\prime}} \rightarrow 0
$$

be a non-trivial extension of $\mathrm{NC}$ deformations over an extension $0 \rightarrow R / M \rightarrow$ $R \rightarrow R^{\prime} \rightarrow 0$ of parameter algebras. Since $\operatorname{Ext}^{1}\left(\mathcal{O}_{X}, \mathcal{O}_{Y}\right)=0$, the natural homomorphism $R \otimes \mathcal{O}_{X} \rightarrow\left(R^{\prime} \otimes \mathcal{O}_{X}\right) / J_{R^{\prime}}$ lifts to an $\mathcal{O}_{X}$-homomorphism $R \otimes \mathcal{O}_{X} \rightarrow E$. Thus there is a commutative diagram

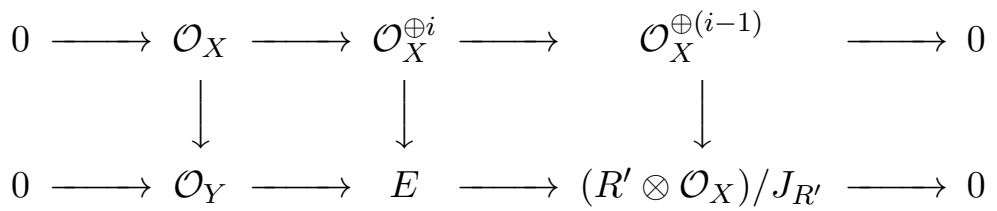

of $\mathcal{O}_{X}$-modules. Since the vertical arrows at both ends are surjective, so is the middle vertical arrow.

Since $H^{0}\left(X, \mathcal{O}_{X}\right) \cong H^{0}\left(X, \mathcal{O}_{Y}\right) \cong k$, the natural homomorphism

$$
H^{0}\left(\mathcal{O}_{X}^{\oplus i}\right) \rightarrow H^{0}(E) \cong R
$$


Non-commutative formal deformations

is an isomorphism. Using this isomorphism, we define a left $R$-module structure on $\mathcal{O}_{X}^{\oplus i}$. Then the middle vertical arrow becomes a homomorphism of left $R \otimes \mathcal{O}_{X}$-modules, and we have $E \cong\left(R \otimes \mathcal{O}_{X}\right) / J_{R}$ for a left $R \otimes \mathcal{O}_{X}$-ideal $J_{R}$.

We prove that the generating sections of $J \otimes \mathcal{O}_{X}(1)$ extend to generating sections of $J_{R} \otimes \mathcal{O}_{X}(1)$ by induction again. From an exact sequence of kernels

$$
0 \rightarrow J \otimes \mathcal{O}_{X}(1) \rightarrow J_{R} \otimes \mathcal{O}_{X}(1) \rightarrow J_{R^{\prime}} \otimes \mathcal{O}_{X}(1) \rightarrow 0
$$

we deduce that the homomorphism $H^{0}\left(X, J_{R} \otimes \mathcal{O}_{X}(1)\right) \rightarrow H^{0}\left(X, J_{R^{\prime}} \otimes\right.$ $\left.\mathcal{O}_{X}(1)\right)$ is surjective, hence the global sections are liftable. By Nakayama's lemma, they are generating.

The following lemma says that the NC deformations of a Cartier divisor is not interesting:

Lemma 7.7. Let $F=\mathcal{O}_{D}$ be the structure sheaf of a Cartier divisor $D \subset X$. Assume that $H^{2}\left(\mathcal{O}_{D}\right)=H^{1}\left(\mathcal{O}_{D}(D)\right)=0$. Then the $N C$ deformations of $F$ are unobstructed, i.e., the deformation ring is isomorphic to a non-commutative formal power series ring $k\left\langle\left\langle x_{1}, \ldots, x_{m}\right\rangle\right\rangle$ for $m=\operatorname{dim} \operatorname{Ext}^{1}(F, F)$.

Proof. We have an exact sequence $0 \rightarrow \mathcal{O}_{X}(-D) \rightarrow \mathcal{O}_{X} \rightarrow \mathcal{O}_{D} \rightarrow 0$. Then there is an exact sequence

$$
\operatorname{Ext}^{1}\left(\mathcal{O}_{X}(-D), F\right) \rightarrow \operatorname{Ext}^{2}(F, F) \rightarrow \operatorname{Ext}^{2}\left(\mathcal{O}_{X}, F\right) .
$$

Hence $\operatorname{Ext}^{2}(F, F) \cong 0$.

Therefore we consider NC deformations of higher codimensional subvarieties:

Example 7.8. Let $X=\mathbf{P}^{n}$ be a projective space with homogeneous coordinates $\left[x_{1}, \ldots, x_{n+1}\right]$, and let $F=\mathcal{O}_{L}=k\left[x_{1}, \ldots, x_{n+1}\right] /\left(x_{1}, \ldots, x_{n-1}\right)^{r}$ be the structure sheaf of a line $L$, where ${ }^{\sim}$ denotes a coherent sheaf on $X$ associated to a graded module.

We claim that the deformation algebra is given by

$\hat{R}=k\left\langle\left\langle a_{1}, b_{1}, \ldots, a_{n-1}, b_{n-1}\right\rangle\right\rangle /\left(a_{i} a_{j}-a_{j} a_{i}, b_{i} b_{j}-b_{j} b_{i}, a_{i} b_{j}-b_{j} a_{i}-a_{j} b_{i}+b_{i} a_{j}\right)$.

and the versal deformation is given as a quotient by a left ideal:

$$
\hat{F}=\hat{R}\left[x_{1}, \ldots, x_{n+1}\right] /\left(x_{1}+a_{1} x_{n}+b_{1} x_{n+1}, \ldots, x_{n-1}+a_{n-1} x_{n}+b_{n-1} x_{n+1}\right)^{r}
$$

where ${ }^{\sim}$ denotes a coherent $\hat{R} \otimes \mathcal{O}_{X}$-module associated to a graded module.

We use Lemma 7.6. The sheaf $J \otimes \mathcal{O}_{X}(1)$ for the ideal sheaf $J$ of $L \subset X$ is generated by global sections $x_{1}, \ldots, x_{n-1}$ and $H^{1}\left(X, J \otimes \mathcal{O}_{X}(1)\right)=0$. Hence $F_{R}$ should be of the form $\left(R \otimes \mathcal{O}_{X}\right) / J_{R}$ for an ideal sheaf $J_{R}$ such that $J_{R} \otimes \mathcal{O}_{X}(1)$ is generated by the following global sections which are linear forms on the $x_{i}$ :

$$
x_{1}+a_{1} x_{n}+b_{1} x_{n+1}, \ldots, x_{n-1}+a_{n-1} x_{n}+b_{n-1} x_{n+1}
$$


Non-commutative formal deformations

where we note that elements of the form $1+r$ with $r \in M$ are invertible, so that the coefficients can be reduced to the above form.

Let $\hat{R}^{\text {ab }}$ be the maximal abelian quotient of $\hat{R}$. Then it is the completed local ring of a Grassmann variety $G(2, n+1)$ at a point. Since $\hat{R}$ and $\hat{R}^{\text {ab }}$ have the same Zariski cotangent spaces, the variables of $\hat{R}$ are the $a_{i}, b_{i}$ as in the above expression of $\hat{F}$. Since $\hat{R}^{\text {ab }}$ is a smooth commutative ring, the relations for $\hat{R}$ are contained in the commutator ideal of the variables.

In order to determine the quadratic terms in the relations, we calculate

$$
m_{2}: \operatorname{Ext}^{1}(F, F) \times \operatorname{Ext}^{1}(F, F) \rightarrow \operatorname{Ext}^{2}(F, F)
$$

explicitly. We have

$$
\begin{aligned}
& \operatorname{Ext}^{1}(F, F) \cong k^{2(n-1)} \cong H^{0}\left(N_{L / X}\right) \\
& \operatorname{Ext}^{2}(F, F) \cong k^{3(n-1)(n-2) / 2} \neq H^{1}\left(N_{L / X}\right)=0
\end{aligned}
$$

where $N_{L / X}$ is the normal bundle of $L$ in $X$. Let $t_{1}, t_{2}$ be the homogeneous coordinates on $L$, and let $\nu_{1}, \ldots, \nu_{n-1}$ be the normal directions of $L$. Then $\operatorname{Ext}^{1}(F, F)$ has a basis $v_{i j}=t_{1}^{i} t_{2}^{1-i} \nu_{j}(i=0,1,1 \leq j \leq n-1)$, and $\operatorname{Ext}^{2}(F, F)$ has a basis $w_{i j k}=t_{1}^{i} t_{2}^{2-i} \nu_{j} \wedge \nu_{k}(i=0,1,2,1 \leq j<k \leq n-1)$. Therefore $m_{2}$ is surjective and its kernel has a basis

$$
\begin{aligned}
& v_{0 j} v_{0 j}, v_{0 j} v_{1 j}, v_{1 j} v_{0 j}, v_{1 j} v_{1 j}, v_{0 j} v_{0 k}+v_{0 k} v_{0 j}, \\
& v_{0 j} v_{1 k}+v_{1 k} v_{0 j}, v_{1 j} v_{0 k}+v_{0 k} v_{1 j}, v_{1 j} v_{1 k}+v_{1 k} v_{1 j}, v_{0 j} v_{1 k}-v_{1 j} v_{0 k}
\end{aligned}
$$

where $1 \leq j \leq n-1$ for the first 4 terms, and $1 \leq j<k \leq n-1$ for the rest. The dual basis of $\operatorname{Im}\left(m_{2}^{*}\right)=\operatorname{Ker}\left(m_{2}\right)^{\perp} \subset\left(\operatorname{Ext}^{1}(F, F)^{*}\right)^{\otimes 2}$ is given by

$$
a_{i} a_{j}-a_{j} a_{i}, b_{i} b_{j}-b_{j} b_{i}, a_{i} b_{j}-b_{j} a_{i}-a_{j} b_{i}+b_{i} a_{j}
$$

for $1 \leq i<j \leq n-1$, where $\left\{a_{i}, b_{j}\right\}_{i, j} \subset \operatorname{Ext}^{1}(F, F)^{*}$ is the dual basis of $\left\{v_{0 i}, v_{1 j}\right\}_{i, j}$. They are the leading terms of the relations for $\hat{R}$.

Now we prove that there are no higher order terms in the relations, i.e., we prove that there is no higher Massey products. We use the fact that the variables $x_{1}, \ldots, x_{n+1}$ in $\hat{F}$ are commutative. We have in $\hat{F}$,

$$
\begin{aligned}
& 0=x_{i} x_{j}-x_{j} x_{i} \\
& =\left(a_{i} a_{j}-a_{j} a_{i}\right) x_{n}^{2}+\left(b_{i} b_{j}-b_{j} b_{i}\right) x_{n+1}^{2}+\left(a_{i} b_{j}-b_{j} a_{i}-a_{j} b_{i}+b_{i} a_{j}\right) x_{n} x_{n+1} .
\end{aligned}
$$

Therefore we have

$$
a_{i} a_{j}-a_{j} a_{i}=b_{i} b_{j}-b_{j} b_{i}=a_{i} b_{j}-b_{j} a_{i}-a_{j} b_{i}+b_{i} a_{j}=0
$$

in $\hat{F}$. If there were higher order terms in the relations of $\hat{R}$ on top of the quadratic relations above, then there were more relations of order $\geq 3$, a contradiction to the fact that the relations are given by $m^{*} \operatorname{Ext}^{2}(F, F)$, and their number is $3(n-1)(n-2) / 2$. Thus the claim is proved.

In particular, if $n \geq 3$, then the NC deformations of $F$ are obstructed, because there are non-trivial relations for $\hat{R}$, but there are more NC deformations than commutative deformations. 
Non-commutative formal deformations

For example, if $n=3$, then lines on $\mathbf{P}^{3}$ are parametrized by $G(2,4)$ under commutative deformations, but the deformation ring for $\mathrm{NC}$ deformations is

$$
\hat{R}=k\langle\langle a, b, c, d\rangle\rangle /(a b-b a, c d-d c, a d-d a-b c+c b) .
$$

We note that this kind of examples are not artificial. For example, if we consider a Calabi-Yau manifold $Y$ such that $L \subset Y \subset \mathbf{P}^{n}$, then the deformation ring of $\mathcal{O}_{L}$ on $Y$, which is an important invariant of an analytic neighborhood of $L$ in $Y$, is a quotient ring of $\hat{R}$ (cf. [2]). In this sense, it is interesting to calculate versal deformations of rational normal curves of higher degrees. 
Non-commutative formal deformations

Example 7.9. Let $X=\mathbf{P}^{4}$ with homogeneous coordinates $[x, y, z, w]$, and let $F=\mathcal{O}_{L}=k[x, y, z, w, t] /\left(x, y, z t-w^{2}\right)^{r}$ for a conic $L$ in $X$.

We claim that the deformation ring $\hat{R}$ of $F$ is given by

$$
\begin{aligned}
& \hat{R}=k\left\langle\left\langle a_{0}, a_{1}, a_{2}, b_{0}, b_{1}, b_{2}, c_{0}, \ldots, c_{4}\right\rangle\right\rangle /\left(a_{0} b_{0}-b_{0} a_{0}+\left(a_{1} b_{1}-b_{1} a_{1}\right) c_{0},\right. \\
& a_{0} b_{1}-b_{1} a_{0}+a_{1} b_{0}-b_{0} a_{1}+\left(a_{1} b_{1}-b_{1} a_{1}\right) c_{1}, \\
& a_{0} b_{2}-b_{2} a_{0}+a_{1} b_{1}-b_{1} a_{1}+a_{2} b_{0}-b_{0} a_{2}+\left(a_{1} b_{1}-b_{1} a_{1}\right) c_{2}, \\
& a_{1} b_{2}-b_{2} a_{1}+a_{2} b_{1}-b_{1} a_{2}+\left(a_{1} b_{1}-b_{1} a_{1}\right) c_{3} \text {, } \\
& a_{2} b_{2}-b_{2} a_{2}+\left(a_{1} b_{1}-b_{1} a_{1}\right) c_{4}, \\
& a_{0} c_{0}-c_{0} a_{0}+\left(a_{1} c_{1}-c_{1} a_{1}\right) c_{0} \text {, } \\
& a_{0} c_{1}-c_{1} a_{0}+a_{1} c_{0}-c_{0} a_{1}+\left(a_{1} c_{1}-c_{1} a_{1}\right) c_{1}, \\
& a_{0} c_{2}-c_{2} a_{0}+a_{1} c_{1}-c_{1} a_{1}+a_{2} c_{0}-c_{0} a_{2}+\left(a_{1} c_{1}-c_{1} a_{1}\right) c_{2}+\left(a_{1} c_{3}-c_{3} a_{1}\right) c_{0}, \\
& a_{0} c_{3}-c_{3} a_{0}+a_{1} c_{2}-c_{2} a_{1}+a_{2} c_{1}-c_{1} a_{2}+\left(a_{1} c_{1}-c_{1} a_{1}\right) c_{3}+\left(a_{1} c_{3}-c_{3} a_{1}\right) c_{1}, \\
& a_{0} c_{4}-c_{4} a_{0}+a_{1} c_{3}-c_{3} a_{1}+a_{2} c_{2}-c_{2} a_{2}+\left(a_{1} c_{1}-c_{1} a_{1}\right) c_{4}+\left(a_{1} c_{3}-c_{3} a_{1}\right) c_{2} \text {, } \\
& a_{1} c_{4}-c_{4} a_{1}+a_{2} c_{3}-c_{3} a_{2}+\left(a_{1} c_{3}-c_{3} a_{1}\right) c_{3} \text {, } \\
& a_{2} c_{4}-c_{4} a_{2}+\left(a_{1} c_{3}-c_{3} a_{1}\right) c_{4} \text {, } \\
& b_{0} c_{0}-c_{0} b_{0}+\left(b_{1} c_{1}-c_{1} b_{1}\right) c_{0}, \\
& b_{0} c_{1}-c_{1} b_{0}+b_{1} c_{0}-c_{0} b_{1}+\left(b_{1} c_{1}-c_{1} b_{1}\right) c_{1} \\
& b_{0} c_{2}-c_{2} b_{0}+b_{1} c_{1}-c_{1} b_{1}+b_{2} c_{0}-c_{0} b_{2}+\left(b_{1} c_{1}-c_{1} b_{1}\right) c_{2}+\left(b_{1} c_{3}-c_{3} b_{1}\right) c_{0}, \\
& b_{0} c_{3}-c_{3} b_{0}+b_{1} c_{2}-c_{2} b_{1}+b_{2} c_{1}-c_{1} b_{2}+\left(b_{1} c_{1}-c_{1} b_{1}\right) c_{3}+\left(b_{1} c_{3}-c_{3} b_{1}\right) c_{1}, \\
& b_{0} c_{4}-c_{4} b_{0}+b_{1} c_{3}-c_{3} b_{1}+b_{2} c_{2}-c_{2} b_{2}+\left(b_{1} c_{1}-c_{1} b_{1}\right) c_{4}+\left(b_{1} c_{3}-c_{3} b_{1}\right) c_{2} \text {, } \\
& b_{1} c_{4}-c_{4} b_{1}+b_{2} c_{3}-c_{3} b_{2}+\left(b_{1} c_{3}-c_{3} b_{1}\right) c_{3} \text {, } \\
& \left.b_{2} c_{4}-c_{4} b_{2}+\left(b_{1} c_{3}-c_{3} b_{1}\right) c_{4}\right)
\end{aligned}
$$

and the versal deformation $\hat{F}$ is given by

$$
\begin{aligned}
& \hat{F}=R[x, y, z, w, t] /\left(x+a_{0} z+a_{1} w+a_{2} t, y+b_{0} z+b_{1} w+b_{2} t\right. \\
& \left.z t-w^{2}+c_{0} z^{2}+c_{1} z w+c_{2} z t+c_{3} w t+c_{4} t^{2}\right) .
\end{aligned}
$$

We note that there are order 3 terms in the relations of $\hat{R}$, i.e., $m_{3} \neq 0$, but $m_{i}=0$ for $i \geq 4$.

In order to prove the claim, we argue similarly to the previous example. We have $N_{L / \mathbf{P}^{4}} \cong \mathcal{O}(2)^{2} \oplus \mathcal{O}(4)$, and

$0 \rightarrow \mathcal{O}_{X}(-4) \rightarrow \mathcal{O}_{X}(-3)^{2} \oplus \mathcal{O}_{X}(-2) \rightarrow \mathcal{O}_{X}(-1)^{2} \oplus \mathcal{O}_{X}(-2) \rightarrow \mathcal{O}_{X} \rightarrow \mathcal{O}_{L} \rightarrow 0$.

Hence

$$
\begin{aligned}
& \operatorname{Ext}^{1}(F, F)=H^{0}\left(\mathbf{P}^{1}, \mathcal{O}(2)^{2} \oplus \mathcal{O}(4)\right) \cong k^{11} \\
& \operatorname{Ext}^{2}(F, F)=H^{0}\left(\mathbf{P}^{1}, \mathcal{O}(4) \oplus \mathcal{O}(6)^{2}\right) \cong k^{19} .
\end{aligned}
$$


Non-commutative formal deformations

$\hat{F}$ is written in the above form by Lemma 7.6. We will determine the relations among variables $a_{i}, b_{j}, c_{k}$ in $\hat{R}$.

The quadratic terms of the relations are determined by the multiplication

$$
m_{2}: \operatorname{Ext}^{1}\left(\mathcal{O}_{L}, \mathcal{O}_{L}\right) \otimes \operatorname{Ext}^{1}\left(\mathcal{O}_{L}, \mathcal{O}_{L}\right) \rightarrow \operatorname{Ext}^{2}\left(\mathcal{O}_{L}, \mathcal{O}_{L}\right)
$$

We take the dual basis

$$
a_{0}^{*}, a_{1}^{*}, a_{2}^{*}, b_{0}^{*}, b_{1}^{*}, b_{2}^{*}, c_{0}^{*}, c_{1}^{*}, c_{2}^{*}, c_{3}^{*}, c_{4}^{*}
$$

of $\operatorname{Ext}^{1}\left(\mathcal{O}_{L}, \mathcal{O}_{L}\right)$, and a basis

$$
d_{0}^{*}, d_{1}^{*}, d_{2}^{*}, d_{3}^{*}, d_{4}^{*}, e_{0}^{*}, e_{1}^{*}, e_{2}^{*}, e_{3}^{*}, e_{4}^{*}, e_{5}^{*}, e_{6}^{*}, f_{0}^{*}, f_{1}^{*}, f_{2}^{*}, f_{3}^{*}, f_{4}^{*}, f_{5}^{*}, f_{6}^{*}
$$

of $\operatorname{Ext}^{2}\left(\mathcal{O}_{L}, \mathcal{O}_{L}\right)$, so that the multiplication map satisfies the following:

$$
\begin{aligned}
& m_{2}\left(a_{i}^{*}, a_{j}^{*}\right)=m_{2}\left(b_{i}^{*}, b_{j}^{*}\right)=m_{2}\left(c_{i}^{*}, c_{j}^{*}\right)=0 \\
& m_{2}\left(a_{i}^{*}, b_{j}^{*}\right)=-m_{2}\left(b_{j}^{*}, a_{i}^{*}\right)=d_{i+j}^{*} \\
& m_{2}\left(a_{i}^{*}, c_{j}^{*}\right)=-m_{2}\left(c_{j}^{*}, a_{i}^{*}\right)=e_{i+j}^{*} \\
& m_{2}\left(b_{i}^{*}, c_{j}^{*}\right)=-m_{2}\left(c_{j}^{*}, b_{i}^{*}\right)=f_{i+j}^{*} .
\end{aligned}
$$

Therefore the image of the map $m_{2}^{*}: \operatorname{Ext}^{2}\left(\mathcal{O}_{L}, \mathcal{O}_{L}\right)^{*} \rightarrow\left(\operatorname{Ext}^{1}\left(\mathcal{O}_{L}, \mathcal{O}_{L}\right)^{*}\right)^{\otimes 2}$ is spanned by the following:

$$
\begin{aligned}
& a_{0} b_{0}-b_{0} a_{0}, a_{0} b_{1}-b_{1} a_{0}+a_{1} b_{0}-b_{0} a_{1}, a_{0} b_{2}-b_{2} a_{0}+a_{1} b_{1}-b_{1} a_{1}+a_{2} b_{0}-b_{0} a_{2}, \\
& a_{1} b_{2}-b_{2} a_{1}+a_{2} b_{1}-b_{1} a_{2}, a_{2} b_{2}-b_{2} a_{2}, \\
& a_{0} c_{0}-c_{0} a_{0}, a_{0} c_{1}-c_{1} a_{0}+a_{1} c_{0}-c_{0} a_{1}, a_{0} c_{2}-c_{2} a_{0}+a_{1} c_{1}-c_{1} a_{1}+a_{2} c_{0}-b_{0} c_{2}, \\
& a_{0} c_{3}-c_{3} a_{0}+a_{1} c_{2}-c_{2} a_{1}+a_{2} c_{1}-c_{1} a_{2}, a_{0} c_{4}-c_{4} a_{0}+a_{1} c_{3}-c_{3} a_{1}+a_{2} c_{2}-c_{2} a_{2}, \\
& a_{1} c_{4}-c_{4} a_{1}+a_{2} c_{3}-c_{3} a_{2}, a_{2} c_{4}-c_{4} a_{2}, \\
& b_{0} c_{0}-c_{0} b_{0}, b_{0} c_{1}-c_{1} b_{0}+b_{1} c_{0}-c_{0} b_{1}, b_{0} c_{2}-c_{2} b_{0}+b_{1} c_{1}-c_{1} b_{1}+b_{2} c_{0}-c_{0} b_{2}, \\
& b_{0} c_{3}-c_{3} b_{0}+b_{1} c_{2}-c_{2} b_{1}+b_{2} c_{1}-c_{1} b_{2}, b_{0} c_{4}-c_{4} b_{0}+b_{1} c_{3}-c_{3} b_{1}+b_{2} c_{2}-c_{2} b_{2}, \\
& b_{1} c_{4}-c_{4} b_{1}+b_{2} c_{3}-c_{3} b_{2}, b_{2} c_{4}-c_{4} b_{2} .
\end{aligned}
$$

These terms give the relations in degree 2 .

The higher order terms are determined by the following argument. We have

$$
\begin{aligned}
& 0=x y-y x \\
& =\left(a_{0} z+a_{1} w+a_{2} t\right)\left(b_{0} z+b_{1} w+b_{2} t\right)-\left(b_{0} z+b_{1} w+b_{2} t\right)\left(a_{0} z+a_{1} w+a_{2} t\right) \\
& =\left(a_{0} b_{0}-b_{0} a_{0}\right) z^{2}+\left(a_{0} b_{1}-b_{1} a_{0}+a_{1} b_{0}-b_{0} a_{1}\right) z w \\
& +\left\{\left(a_{0} b_{2}-b_{2} a_{0}+a_{2} b_{0}-b_{0} a_{2}\right) z t+\left(a_{1} b_{1}-b_{1} a_{1}\right) w^{2}\right\} \\
& +\left(a_{1} b_{2}-b_{2} a_{1}+a_{2} b_{1}-b_{1} a_{2}\right) w t+\left(a_{2} b_{2}-b_{2} a_{2}\right) t^{2} \\
& 21
\end{aligned}
$$


Non-commutative formal deformations

and

$$
\begin{aligned}
& 0=x\left(z t-w^{2}\right)-\left(z t-w^{2}\right) x \\
& =\left(a_{0} z+a_{1} w+a_{2} t\right)\left(z t-w^{2}+c_{0} z^{2}+c_{1} z w+c_{2} z t+c_{3} w t+c_{4} t^{2}\right) \\
& -\left(z t-w^{2}+c_{0} z^{2}+c_{1} z w+c_{2} z t+c_{3} w t+c_{4} t^{2}\right)\left(x+a_{0} z+a_{1} w+a_{2} t\right) \\
& =\left(a_{0} c_{0}-c_{0} a_{0}\right) z^{3}+\left(a_{0} c_{1}-c_{1} a_{0}+a_{1} c_{0}-c_{0} a_{1}\right) z^{2} w \\
& +\left\{\left(a_{0} c_{2}-c_{2} a_{0}+a_{2} c_{0}-c_{0} a_{2}\right) z^{2} t+\left(a_{1} c_{1}-c_{1} a_{1}\right) z w^{2}\right\} \\
& +\left(a_{0} c_{3}-c_{3} a_{0}+a_{1} c_{2}-c_{2} a_{1}+a_{2} c_{1}-c_{1} a_{2}\right) z w t \\
& +\left\{\left(a_{0} c_{4}-c_{4} a_{0}+a_{2} c_{2}-c_{2} a_{2}\right) z t^{2}+\left(a_{1} c_{3}-c_{3} a_{1}\right) w^{2} t\right\} \\
& +\left(a_{1} c_{4}-c_{4} a_{1}+a_{2} c_{3}-c_{3} a_{2}\right) w t^{2}+\left(a_{2} c_{4}-c_{4} a_{2}\right) t^{3} .
\end{aligned}
$$

Since

$$
\begin{aligned}
& \left(a_{1} b_{1}-b_{1} a_{1}\right) w^{2} \equiv\left(a_{1} b_{1}-b_{1} a_{1}\right)\left(z t+c_{0} z^{2}+c_{1} z w+c_{2} z t+c_{3} w t+c_{4} t^{2}\right) \\
& \left(a_{1} c_{1}-c_{1} a_{1}\right) z w^{2} \equiv\left(a_{1} c_{1}-c_{1} a_{1}\right)\left(z^{2} t+c_{0} z^{3}+c_{1} z^{2} w+c_{2} z^{2} t+c_{3} z w t+c_{4} z t^{2}\right) \\
& \left(a_{1} c_{3}-c_{3} a_{1}\right) w^{2} t \equiv\left(a_{1} c_{3}-c_{3} a_{1}\right)\left(z t^{2}+c_{0} z^{2} t+c_{1} z w t+c_{2} z t^{2}+c_{3} w t^{2}+c_{4} t^{3}\right)
\end{aligned}
$$

modulo $\left(z t-w^{2}+c_{0} z^{2}+c_{1} z w+c_{2} z t+c_{3} w t+c_{4} t^{2}\right)$, (7.1) and (7.2) become

$$
\begin{aligned}
& \left(a_{0} b_{0}-b_{0} a_{0}+\left(a_{1} b_{1}-b_{1} a_{1}\right) c_{0}\right) z^{2} \\
& +\left(a_{0} b_{1}-b_{1} a_{0}+a_{1} b_{0}-b_{0} a_{1}+\left(a_{1} b_{1}-b_{1} a_{1}\right) c_{1}\right) z w \\
& +\left(a_{0} b_{2}-b_{2} a_{0}+a_{1} b_{1}-b_{1} a_{1}+a_{2} b_{0}-b_{0} a_{2}+\left(a_{1} b_{1}-b_{1} a_{1}\right) c_{2}\right) z t \\
& +\left(a_{1} b_{2}-b_{2} a_{1}+a_{2} b_{1}-b_{1} a_{2}+\left(a_{1} b_{1}-b_{1} a_{1}\right) c_{3}\right) w t \\
& +\left(a_{2} b_{2}-b_{2} a_{2}+\left(a_{1} b_{1}-b_{1} a_{1}\right) c_{4}\right) t^{2}
\end{aligned}
$$

and

$$
\begin{aligned}
& \left(a_{0} c_{0}-c_{0} a_{0}+\left(a_{1} c_{1}-c_{1} a_{1}\right) c_{0}\right) z^{3} \\
& +\left(a_{0} c_{1}-c_{1} a_{0}+a_{1} c_{0}-c_{0} a_{1}+\left(a_{1} c_{1}-c_{1} a_{1}\right) c_{1}\right) z^{2} w \\
& +\left(a_{0} c_{2}-c_{2} a_{0}+a_{1} c_{1}-c_{1} a_{1}+a_{2} c_{0}-c_{0} a_{2}+\left(a_{1} c_{1}-c_{1} a_{1}\right) c_{2}+\left(a_{1} c_{3}-c_{3} a_{1}\right) c_{0}\right) z^{2} t \\
& +\left(a_{0} c_{3}-c_{3} a_{0}+a_{1} c_{2}-c_{2} a_{1}+a_{2} c_{1}-c_{1} a_{2}+\left(a_{1} c_{1}-c_{1} a_{1}\right) c_{3}+\left(a_{1} c_{3}-c_{3} a_{1}\right) c_{1}\right) z w t \\
& +\left(a_{0} c_{4}-c_{4} a_{0}+a_{1} c_{3}-c_{3} a_{1}+a_{2} c_{2}-c_{2} a_{2}+\left(a_{1} c_{1}-c_{1} a_{1}\right) c_{4}+\left(a_{1} c_{3}-c_{3} a_{1}\right) c_{2}\right) z t^{2} \\
& +\left(a_{1} c_{4}-c_{4} a_{1}+a_{2} c_{3}-c_{3} a_{2}+\left(a_{1} c_{3}-c_{3} a_{1}\right) c_{3}\right) w t^{2} \\
& +\left(a_{2} c_{4}-c_{4} a_{2}+\left(a_{1} c_{3}-c_{3} a_{1}\right) c_{4}\right) t^{3} .
\end{aligned}
$$

Therefore we have our claim.

Remark 7.10. Let $C$ be a smooth rational curve on a Calabi-Yau 3-fold. If $C$ is contractible to a point by a bimeromorphic morphism $X \rightarrow \bar{X}$ whose exceptional locus coincides with $C$, then the $\mathrm{NC}$ deformation ring of $\mathcal{O}_{C}$ is finite dimensional. It is interesting to know whether the converse is true.

By [1], there is an example where $C$ is not contractible but the abelianization of the deformation ring is finite dimensional. In this example, the 
Non-commutative formal deformations

normal bundle of $C$ is isomorphic to $\mathcal{O}(2) \oplus \mathcal{O}(-4)$ (hence not contractible). The deformation ring is a quotient of a non-commutative formal power series ring with 3 variables by an ideal generated by 3 relations. By [3] and [13], it is known that such a ring is finite dimensional if the 3 relations are generic quadratic forms (this information, opposite to author's naive expectation, was given to the author by Professor Spela Spenko through Professors Michel Van den Bergh and Keiji Oguiso).

Remark 7.11. By [12] Lemma 4.1, the versal formal NC deformation is convergent in the sense that $\left\|m_{n}\right\|<C^{n}$ and $\left\|f_{n}\right\|<C^{n}$ for suitable norms and a constant $C>0$ which is independent of $n$.

Remark 7.12. Zheng Hua informed the author that, if the bounded derived category of coherent sheaves $D^{b}(\operatorname{coh}(X))$ has a strong exceptional collection consisting of line bundles, e.g., $X \cong \mathbf{P}^{n}$, then the NC deformation algebra of any coherent sheaf $F$ on $X$ is algebraic in the following sense; the $A^{\infty}$ algebra $\operatorname{Ext}^{*}(F, F)$ is quasi-isomorphic to a finite dimensional $A^{\infty}$-algebra $B$ such that $m_{n}^{B}=0$ for all $n \geq n_{0}$ with a fixed $n_{0}$ (cf. [4] Theorem 4.4, [5]). We note that $B$ is not necessarily minimal, i.e., $m_{1}^{B}$ may not vanish.

\section{REFERENCES}

[1] Clemens, H. The infinitesimal Abel-Jacobi mapping and moving the $O(2)+O(-4)$ curve. Duke Math. J. 59-1 (1989), 233-240.

[2] Donovan, W.; Wemyss, M. Noncommutative deformations and flops. Duke Math. J., 165-8 (2016), 1397-1474.

[3] Golod, E. S.; ShafarevichI, R. On the class field tower. Izvestiya of the Academy of Sciences USSR, a series of Mat., 28 (1964), 261-272.

[4] Hua, Zheng. Chern-Simons functions on toric Calabi-Yau threefolds and DonaldsonThomas theory. Pacific J. Math. 277 (2015), 119-147.

[5] Hua, Zheng. Algebraicity of noncommutative deformation. preprint.

[6] Kawamata, Yujiro. On multi-pointed non-commutative deformations and Calabi-Yau threefolds. Compositio Math. 154 (2018), 1815-1842. doi:10.1112/S0010437X18007248.

[7] Kawamata, Yujiro. Semi-orthogonal decomposition of a derived category of a 3-fold with an ordinary double point. arXiv:1903.00801.

[8] Kadeishvili, T.V. On the homology theory of fiber spaces. Uspekhi Mat. Nauk 35:3 (1980), 183-188. Russian Math. Surveys, 35:3 (1980), 231-238.

[9] Keller, Bernhard. Introduction to A-infinity algebras and modules. Homology Homotopy Appl., 3-1 (2001), 1-35.

[10] Laudal, O. A. Noncommutative deformations of modules. Homology Homotopy Appl. 4 (2002), 357-396.

[11] Schlessinger, M. Functors of Artin rings. Trans. Amer. Math. Soc. 130 (1968), 208222.

[12] Toda, Yukinobu. Moduli stacks of semistable sheaves and representations of Extquivers. Geometry and Topology, 22 (2018), 3083-3144.

[13] Vinberg, E. B. On the theorem concerning the infinite dimensionality of an associative algebra. Izv. Akad. Nauk SSSR Ser. Mat., 1965, Volume 29, Issue 1, 209-214.

Graduate School of Mathematical Sciences, University of Tokyo, Komaba, Meguro, Tokyo, 153-8914, Japan.

Morningside Center of Mathematics, Chinese Academy of Sciences, Haidian District, Beijing, China 100190 
Non-commutative formal deformations

Department of Mathematical Sciences, Korea Advanced Institute of Science and Technology, 291 Daehak-ro, Yuseong-gu, Daejeon 34141, Korea.

National Center for Theoretical Sciences, Mathematics Division, National Taiwan University, Taipei, 106, Taiwan.

kawamata@ms.u-tokyo.ac.jp 